\title{
Chapter 11 \\ Adjusting the Manner of Language \\ Processing to the Social Context: \\ Attention Allocation During Interactions \\ with Non-native Speakers
}

Shiri Lev-Ari

\subsection{Introduction}

Models of language processing traditionally assume that we process the language of all speakers similarly, regardless of who they are. Thus, they assume that we will understand the sentence $I$ just ate two pieces of pie using the same cues and reaching the same interpretation regardless of whether it was uttered by a young teenager, an elderly woman, our middle-aged neighbour or our non-native-speaking friend. Occasionally, there might be break downs in communication. For example, a language learner might select a suboptimal lexical item, using the sentence above, for example, to describe eating slices of a quiche, a cake or even two brownies. In these cases, we, as listeners, would note the error, and then correct it, inferring the meaning from the context. Importantly, the initial process of constructing a meaning out of the input is implicitly assumed to remain invariable, and the correction takes place after an incompatibility between the linguistic input and the context had been detected. More recent evidence, however, indicates that language processing is not invariable, but, in fact, adjusts to the situation. This evidence shows that the cues that listeners attend to during language processing might change according to the identity of the speaker. This chapter will review such evidence while focusing on evidence that shows that listeners' expectations of non-native speakers lead them to allocate less attention to linguistic cues and greater attention to contextual cues when processing the language of non-native speakers.

S. Lev-Ari $(\bowtie)$

Nijmegen, Netherlands

e-mail: shiri.lev-ari@mpi.nl 


\subsubsection{Expectations and Language Processing}

When we listen to people, we spontaneously generate expectations for what they might say next. In fact, upon hearing the voice of an unfamiliar speaker, we immediately extract from it information about the speaker's gender, age, and socioeconomic status, and this information is sufficient to immediately induce certain expectations about what the speaker is likely to say. Therefore, hearing a voice of a child saying Every evening I drink some wine before I go to sleep leads to increased N400, an ERP component associated with greater difficulty in semantic integration, that is similar in timing and distribution to the one induced by semantic anomalies (Van Berkum et al. 2008).

In fact, the integration of social information is an integral aspect of language processing. For example, the interpretation of a phoneme as $/ \mho /$ or $/ \Lambda /$ depends on whether we see or imagine a man or a woman speaking, as the boundary between the two vowels is at different formant frequencies for men and women (Johnson et al. 1999). Such integration of social information, however, can sometimes lead us astray and distort our perception. For example, it can lead us to perceive the same vowel as a standard /a/ if we believe the speaker is from a region where standard American English is spoken, but as an /aw/ if we believe the speaker is from Canada, where /a/is produced as the raised diphthong /aw/ (Niedzielski 1999).

The role of expectations is not restricted to the stage of the final interpretation of speech. Recent studies show that expectations can influence the very manner of language processing by shifting the weight we give different cues. Such shifting of weights can lead us to avoid making inferences that we commonly do (Arnold et al. 2007; Grodner and Sedivy 2011). For example, while listeners commonly infer from disfluency in the speech that the speaker is about to describe something hard, they no longer make that inference if they believe the speaker suffers from object agnosia, a difficulty with naming objects (Arnold et al. 2007). Similarly, expecting the input to be noisy and therefore unreliable attenuates our tendency to rely on onset versus offset phonetic information (McQueen and Huettig 2012).

\subsection{Processing the Language of Non-native Speakers}

One type of situation in which listeners hold specific expectations about the speaker is the case of processing the language of non-native speakers. Non-native speakers often have lower linguistic proficiency. They may make grammatical errors or suboptimal lexical choices, and we as listeners know and expect that (Hanulikova et al. 2012). Non-native speakers' lower proficiency can render their speech less reliable in conveying their intentions. Therefore, one way for listeners to optimise communication is to attend less to the less reliable linguistic input, and instead attend more to contextual information, which is equally reliable with native and non-native speakers. The remainder of this chapter will focus on this case of processing the language of non-native speakers in order to illustrate the manner in 
which the allocation of attention and resources can adjust to circumstances. It will also survey the consequences of such an adjustment as well as its constraints.

\subsubsection{Good-Enough Representations}

Suppose your friend who had just referred to the quiche as a pie tells you about the wonderful dish he is planning to prepare tonight. Your friend may use specific terms, such as a pie or a quiche, to refer to this dish, but considering his unreliable use of such terms, encoding the message as being about a baked good or a dish might be better, as it would require less effort while potentially increasing accuracy.

Less detailed "good-enough" processing occurs often even when processing the language of native speakers. We often do not process linguistic input in full, but, instead, only process it to a level that is perceived to be sufficient for the situational demands, and then fill-in missing information based on our general knowledge. This can lead us to occasionally not notice anomalies in the input, misrepresent the content of the utterance, or miss content changes in it. Thus we might encode a sentence about a man biting a dog as being about a dog biting a man, because our world knowledge would favour this interpretation of the lessdetailed representation we generated (Ferreira et al. 2002). At other times, we might miss changes in story details such as the fact that a story that earlier mentioned cider being drunk at the pub now describes beer (Sturt et al. 2004).

Importantly, this tendency of ours to process and represent language in less detail depends on our expectations regarding speaker reliability. This leads us to attend even less to the details in the linguistic input when we listen to non-native speakers. This was evidenced by a recent study in which participants needed to detect changes in a story told by either a native or a non-native speaker. In this study, participants first listened to either a native or a non-native speaker tell a story about his friend. Later, they received a surprise memory test, in which they read a modified written version of the story, and were asked to detect changes that were made to the story. The changes were always word substitutions to semantically related words. For example, a statement by the speaker that his friend never has the time to cook changed to a statement declaring that she never has the patience to cook. As predicted, participants who listened to a non-native speaker tell the story detected fewer changes than those who listened to a native speaker (Lev-Ari and Keysar 2012). One may wonder whether it is simply because nonnative speakers are harder to understand, and because processing their speech imposes greater cognitive load. The study, however, had two listening conditions that manipulated the relevance of participants' communicative expectations. When participants' goal was to listen for comprehension in anticipation of later answering comprehension questions, participants showed the aforementioned difference in memory for the language of the native compared with the non-native speaker. In the other condition, however, where participants were told to memorise the story 
they hear, because they will later be asked to detect word changes, participants showed equal performance with the two speakers. This indicates that we are able to process the language of non-native speakers in detail, but choose to attend to the linguistic details less when listening for comprehension.

This study then shows adjustment in the manner of language processing according to the speaker's characteristics. In general, there is evidence that working memory can influence listeners' and readers' ability to integrate different types of information in a timely manner during language processing (Federmeier and Kutas 2005; Just and Carpenter 1992; Traxler et al. 2005). A follow-up study examined whether this better ability at integrating different types of information leads those with higher working memory to be better able to integrate the information about the speaker in order to better adjust their manner of processing according to whether the speaker is a native or a non-native speaker. Using the same word change detection task, the follow-up study showed that above a certain working memory threshold, higher working memory leads to greater engagement in less-detailed processing, and consequently, to poorer performance with the nonnative speaker. This finding is particularly striking, as higher working memory is often associated with better performance (Conway et al. 2002).

\subsubsection{Attention to Contextual Cues}

Other than representing language in less detail, listeners can also potentially improve communication with non-native speakers by attending more to contextual information when processing the language of non-native speakers, as the reliability of contextual information is unaffected by the proficiency of the speaker. Such greater reliance on contextual information can be reflected in at least two manners: by weighing contextual information more heavily than ordinarily, but also by using it earlier in order to predict what a speaker is about to say. After all, we commonly make such use of contextual information-whether it is the linguistic context, the visual context or the context's affordances- to predict the forthcoming input, and such contextual information can influence both syntactic and lexical interpretation of the linguistic input (Chambersa et al. 2004; Duffy et al. 1988; Tanenhaus et al. 1995). The question is then whether we increase this tendency to rely on contextual information when its reliability is relatively high compared with other sources of information.

To understand how such an increase in reliance might be manifested, consider the following example from one of the experiments that tested for increased reliance when processing the language of non-native speakers. Suppose you sit in front of a screen with pictures, and are asked to follow instructions that will indicate which pictures from the set to select. You are further told that all the pictures that you will be asked to select share something in common. You are then told to select the cookie. Once you select it, you are told to select the cheese, and after you do so as well, you are told to select the tortilla. By this stage, you will have 
likely noted that all the pictures that you had selected were food items. You will therefore expect to next be instructed to select the brownie. If attention to topdown information and reliance on it increase during the processing of non-native language, then you should be more likely to rely on this expectation to be told to click on the brownie if you follow instructions provided by a non-native rather than a native speaker. Such greater reliance on expectations can be indicated, for example, by greater likelihood of fixating on the brownie (the competitor) already at word onset, just before you hear the next instruction.

The next instruction, however, is to select the /par/. One meaning of /par/is pie, which is semantically related to a brownie, as both are food items. Therefore, if listening to non-native speakers leads listeners to attend less to the linguistic details, then you should be less likely to notice that there is a discrepancy between the label,/pai/, and the expected referent, the brownie, if the instructions are provided by a non-native speaker. This would be indicated by lower likelihood of attending to the target object, the symbol $\pi$, which relates to the previous selected pictures by the much less dominant theme of the geometry of a circle (as all previously selected items are round).

Additionally, greater reliance on top-down expectations, whether while noticing the discrepancy of the label or not, should lead to greater likelihood of selecting the brownie rather than the symbol $\pi$.

To recapitulate, participants in this experiment selected pictures that were semantically related to one another according to instructions provided by either a native or a non-native speaker. On critical trials, the first three pictures participants were instructed to select induced an expectation for a specific fourth object (competitor) to be selected. The following critical instruction, however, was always a homophone, which had one meaning that is semantically related to the expected object, yet inappropriate for it (e.g., pie), and one meaning that fits another object on the screen $(\pi)$, whose relation to the first three objects is more tenuous. It was tested whether the participants who listened to the non-native speakers were more likely to allocate attention to the contextually expected competitor object earlier, whether they were more likely to eventually select it, and whether they were less likely to attend to the target object.

Results indeed showed all these patterns of adjustment to non-native speakers (Lev-Ari 2015): In terms of final selection, listeners selected the context-appropriate competitor (brownie) rather than the target $(\pi)$ more often when they followed instructions provided by a non-native speaker than by a native speaker. Listeners were also more likely to look at the contextually appropriate competitor already at word onset and were less likely to look at the target at all if they listened to a nonnative rather than a native speaker, but these effects were modulated by working memory. As with the shift to less-detailed representation in the change detection task described earlier, higher working memory led to greater difference between performance with native and non-native speakers, whereas lower working memory led to a more invariable manner of processing that does not depend on speaker.

The study then illustrates the flexibility of our processing mechanisms and their ability to adjust to the social circumstances. It shows that both the type of cues 
we attend to and the point in time at which we do so can vary according to the expected reliability of the cues. The studies also indicate that this ability to adjust our manner of processing according to the social circumstances is demanding and therefore constrained by our cognitive abilities, such as our working memory.

\subsubsection{Consequences for Other Aspects of Language Processing}

So far, this chapter described the allocation of attention to different cues as an isolated process. Yet the allocation of attention to a specific cue is a sub-process in a complex language processing mechanism that is composed of multiple interrelated components. Consequently, modifications to one aspect of the mechanism can have consequences for other aspects. Such is the case with the lesser attention to linguistic detail in processing. As will be described below, this adjustment in attention allocation impacts lexical competition and access.

Lexical access is achieved by inhibiting semantic competitors, and the more similar a competitor is, the greater effort that is needed to inhibit it (Anderson et al. 1994). Yet similarity is context-dependent. In the case of listening to a nonnative speaker, the lesser attention to linguistic details can lead to greater similarity between items. This is so, because specifying fewer details for each lexical item means that many of the characteristics that distinguish related items from one another are likely to be left unspecified.

One study tested whether the processing of the language of non-native speakers therefore leads to greater lexical competition. The study relied on the RetrievalInduced Inhibition paradigm, also known as the Retrieval-Induced Forgetting (Anderson et al. 1994; Veling and van Knippenberg 2004). In this task, participants memorise words from a few specific categories. The presentation of these words is blocked by category and the words appear next to their category name. Following the memorisation stage, participants do a cued recall task, in which they receive the category name with the first letter of a word they had memorised, and their task is to retrieve that word. Importantly, in this stage, they practice only half of the words from some of the categories, and no words from other categories. Consequently, there are three types of words: practiced words, words that were not practiced but belong to categories that were practiced, and words that were not practiced and belong to categories from which no word had been practiced. Finally, participants perform a recognition test on all memorised words, together with fillers. Practiced words are naturally recognised faster than non-practiced words in this task. The crucial question though is whether there is a difference between the recognition of the two types of words that were not practiced. The exercise of inhibition during lexical access in the cued recall task should lead to the inhibition of the unpracticed words from the practised categories. This should lead those words to be recognised more slowly than the unpracticed words from 
the unpracticed categories, and this difference in recognition should be larger the more inhibition was exercised during cued recall.

Returning to the case of processing the language of non-native speakers, if listeners attend less to linguistic details and thus experience greater lexical competition with non-native speakers, then they should show greater difference between the recognition of the two types of unpracticed words. This is what the study found (Lev-Ari et al. 2011). Participants who earlier listened to a story by a nonnative speaker showed greater lexical competition than those who listened to a story by a native speaker. This is despite the fact that the lexical items whose competition was measured were not produced by the native and non-native speakers but were presented afterwards. Therefore, the words in the two speaker conditions did not differ in any way nor did they require different amounts of processing. The direction of the difference in inhibition-greater inhibition after listening to non-native speakers - also rules out the option that the difference is due to the greater cognitive load involved in processing the language of non-native speakers, as greater cognitive load should lead to the dwindling of resources, and therefore, to lower, rather than higher inhibition. Still, one may wonder whether the greater inhibition following the processing of the language of non-native speakers is linguistic in nature or whether it is due to some change in the general use of inhibition when processing the language of non-native speakers. To examine that possibility, a non-linguistic version of the task, in which participants memorised and practiced novel difficult-to-label shapes and visual arrays, was run. In contrast to the linguistic version, there were no differences in this case between the level of inhibition that those who listened to the native speaker and those who listened to the non-native speaker exhibited. The changes in the exercise of inhibition, then, are linguistic in nature and restricted to language processing mechanisms. The study then shows how changing the allocation of attention to linguistic detail influences lexical competition. It thus illustrates how adapting one aspect of processing to improve communication can lead to unintended changes in other aspects of the language processing mechanisms.

\subsubsection{Consequences for Processing Other Linguistic Input}

Our language processing mechanisms, then, are flexible. As I have shown, the way that attention is allocated-which cues are considered, at what point in time, and how much weight they are assigned-varies according to the nature and demands of the situation. One may wonder, however, how flexible our language processing mechanism is in terms of switching between manners of language processing. For example, do we adjust to each speaker in a multi-party interaction? Furthermore, even when interacting solely with one speaker, a non-native one, are we able to use different manners of processing for her language and for ours? This latter question is particularly intriguing considering models that posit that language production mechanisms are engaged during comprehension of others' language 
(Pickering and Garrod 2007) and that language comprehension mechanisms monitor our own productions (Levelt 1983).

A recent study tested this question by comparing the level of detail in the representation of one's own language during interactions with non-native versus native speakers. Participants in this study were interviewed by a native or non-native speaking confederate about a short story they had both read. After a short distractor task, participants received a surprise memory test, where they had to identify their responses from a list of possible responses. Those who were interviewed by a non-native speaker performed more poorly in this task (Lev-Ari et al. 2011).

Another interesting aspect of this study was the finding that the degree of adjustment to the non-native speaker in production predicted the degree of adjustment in processing. In general, we adjust our productions to the characteristics of our speakers. A particularly well-studied adaptation is the one towards infants. Yet we adjust our production to non-native speakers as well, and one of the phonetic hallmarks of such adjustment is slower speech rate (Uther et al. 2007). Interestingly, in the aforementioned study, those who adjusted their productions the most towards non-native speakers, as indicated by slower speech rate, also showed the greatest adjustment in processing, as reflected in poorer detection of their own responses. Importantly, there was no relation between speech rate and response detection among those who were interviewed by a native speaker, indicating that it is not simply that those who speak slowly have poorer recall.

This study then shows that our language processing mechanism adjusts as a whole: the degree of adjustment in speaking relates to the degree of adjustment in processing and representation, and adjustment to one speaker modifies the processing of the language of other speakers. Thus, when we adjust the way we allocate attention while listening to one speaker, this modified manner of allocation of attention carries over to the processing of other language, even our own.

\subsubsection{Social Consequences}

As we have seen so far, social factors, such as speaker characteristics, influence the allocation of attention during language processing. The relation between allocation of attention and social factors, however, is a two ways street. As linguistic interactions are embedded in social interactions, the studies presented so far suggest that the characteristics of our linguistic interaction partner can influence the degree to which we attend to the context of a social situation. Such differential allocation of attention context can have far reaching consequences, as many social phenomena depend on consideration of top-down contextual information. To take one, perspective taking, the ability to take into account the fact that others hold different information from us, depends on an ability to attend to and integrate common ground. Therefore, the findings that we attend more to the context when interacting with non-native speakers suggest, quite counter-intuitively, that we would be better at perspective taking when interacting with non-native speakers. 


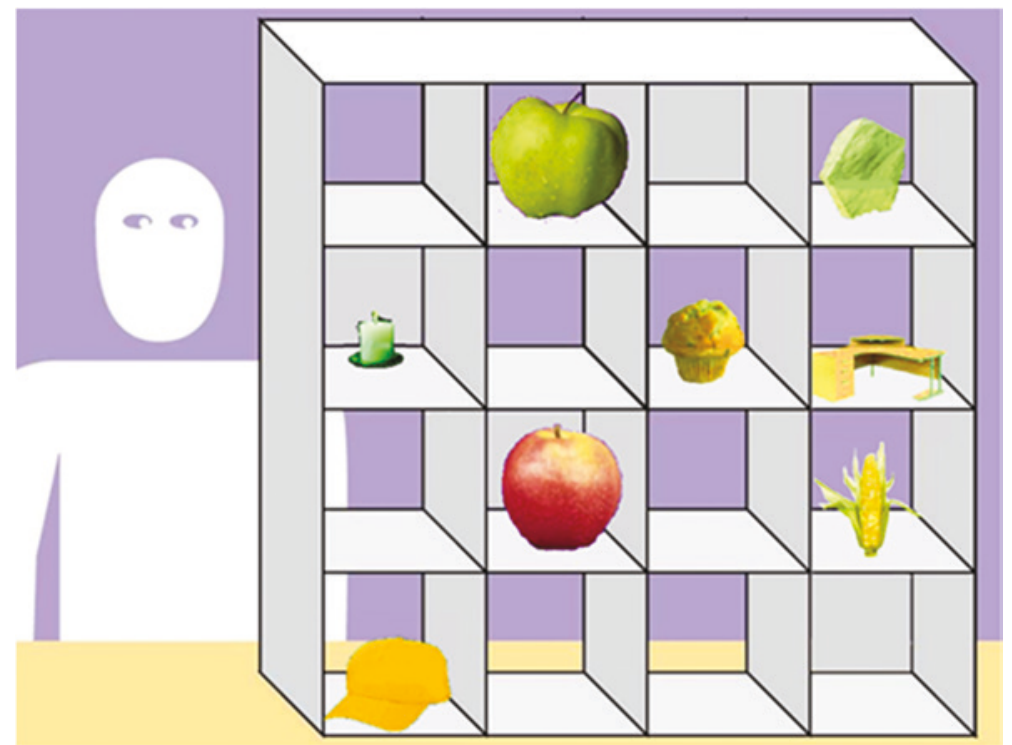

Fig. 11.1 An illustration of a trial in a perspective taking task

One study tested this possibility using a computerised version of the common perspective taking task (Keysar et al. 2000; Dumontheil et al. 2010). In this task, a "director" instructs a participant, sitting on the other side of a grid, to move objects from one cell in the grid into another. Some of the cells of this grid are occluded from the director's point of view but not from the instruction-follower's view (see Fig. 11.1). On experimental trials, the director's instruction, e.g., Put the apple below the muffin, could fit both a mutually visible object (the green apple) and an object occluded from the director's view (the red apple), if the director's perspective is not taken into account. Therefore, greater proportion of looks towards the occluded apple in such trials compared with a control trial, where a non-competing object is placed in the occluded cell, indicates failure to take the director's perspective. A comparison of the gaze pattern of participants who listened to a non-native speaker with those who listened to a native speaker revealed that, indeed, participants were more attuned to the perspective of the director when she was a non-native speaker (Lev-Ari, Barr and Keysar, under review). This effect was found despite the fact that listening to non-native speakers imposes a cognitive load, and added cognitive load in general leads to worse perspective taking (Epley et al. 2004). This study thus shows that adjustment in allocation of attention during language processing can have non-linguistic social consequences. 


\subsection{Summary}

Language processing is a complex mechanism where multiple cues must be attended to and integrated. Importantly, this mechanism operates in a social context. Therefore, the traditional study of it in a decontextualized setting obscures some of its characteristics. One such understudied characteristic is the way that social characteristics, such as an interlocutor's identity, can influence the allocation of attention during language processing.

The studies described here provide strong support that listeners process differently the language of non-native speakers. They further illustrate the manner in which the processing mechanism can adjust itself, namely, by changing the time point at which different cues are attended to and the weight that they are given. The studies also show how an adjustment in allocation of attention can influence other aspects of processing because of the interrelated nature of the processing mechanism. Furthermore, the studies show that adjustments in manner of processing can lead to unexpected social consequences. At the same time, the research demonstrates the constraints of the flexibility of our processing mechanism. For example, it shows that flexibility in attention allocation requires cognitive resources, and that we do not easily re-allocate our attention with frequent changes in the source of the input, such as a switch between speakers. This chapter described one specific unintended linguistic consequence and one social consequence to the adjustment in processing. Needless to say, there could be additional linguistic and social consequences to the adjustment in attention allocation when listening to non-native speakers. Similarly, there could be different adaptations in allocation of attention when interacting with other types of speakers, who induce other types of expectations.

The research described here, then, points to the importance of examining language processing, including its sub-processes such as allocation of attention to different cues, under different social conditions and understanding how cognitive processes are socially mediated.

\section{References}

Anderson, M. C., Bjork, R. A., \& Bjork, E. L. (1994). Remembering can cause forgetting: Retrieval dynamics in long-term memory. Journal of Experimental Psychology. Learning, Memory, and Cognition, 20(5), 1063-1087.

Arnold, J. E., Hudson Kam, C. L., \& Tanenhaus, M. K. (2007). If you say thee uh-you're describing something hard: The on-line attribution of disfluency during reference comprehension. The Journal of Experimental Psychology: Learning, Memory and Cognition, 33, 914-930.

Chambers, C. G., Tanenhaus, M. K., \& Magnuson, J. S. (2004). Actions and affordances in syntactic ambiguity resolution. Journal of Experimental Psychology. Learning, Memory, and Cognition, 30, 687-696.

Conway, A. R. A., Cowan, N., Bunting, M. F., Therriault, D., \& Minkoff, S. (2002). A latent variable analysis of working memory capacity, short term memory capacity, processing speed, and general fluid intelligence. Intelligence, 30, 163-183. 
Duffy, S. A., Morris, R. K., \& Rayner, K. (1988). Lexical ambiguity and fixation times in reading. Journal of Memory and Language, 27, 429-446.

Dumontheil, I., Apperly, I. A., \& Blakemore, S. J. (2010). Online use of mental state inferences continues to develop in late adolescence. Developmental Science, 13(2), 331-338.

Epley, N., van Keysar, B., Boyen, L., \& Gilovich, T. (2004). Perspective Taking as Egocentric Anchoring and Adjustment. Journal of Personality and Social Psychology, 87(3), 327-339.

Federmeier, K. D., \& Kutas, M. (2005). Aging in context: Age-related changes in context use during language comprehension. Psychophysiology, 42, 133-142.

Ferreira, F., Ferraro, V., \& Bailey, K. G. D. (2002). Good-enough representations in language comprehension. Current Directions in Psychological Science, 11, 11-15.

Grodner, D., \& Sedivy, J. C. (2011). The effects of speaker-specific information on Pragmaticinferences. In N. Pearlmutter \& E. Gibson (Eds.), The processing and acquisition of reference (pp. 239-272). Cambridge: MIT Press.

Hanulikova, A., Van Alphen, P. M., Van Goch, M., \& Weber, A. (2012). When one person's mistake is another's standard usage: The effect of foreign accent on syntactic processing. Journal of Cognitive Neuroscience, 24(4), 878-887.

Johnson, K., Strand, E. A., \& D'Imperio, M. (1999). Auditory-visual integration of talker gender in vowel perception. Journal of Phonetics., 27, 359-384.

Just, M. A., \& Carpenter, P. A. (1992). A capacity theory of comprehension: Individual differences in working memory. Psychological Review, 99, 122-149.

Keysar, B., Barr, D. J., Balin, J. A., \& Brauner, J. S. (2000). Taking perspective in conversation: The role of mutual knowledge in comprehension. Psychological Science, 11, 32-38.

Lev-Ari, S. (2015). Comprehending non-native speakers: Theory and evidence for adjustment in manner of processing. Frontiers in Psychology, 5, 1546.

Lev-Ari, S., Barr, D., \& Keysar, B. (under review). Listeners are better attuned to non-native speakers' perspectives.

Lev-Ari, S., Ho, E., \& Keysar, B. (2011). Interacting with non-native speakers induces "goodenough" representation. Poster presented at The $24^{\text {th }}$ Annual CUNY Conference on Human Sentence Processing. CA: Stanford.

Lev-Ari, S., \& Keysar, B. (2012). Less-detailed representation of non-native language: Why nonnative speakers' stories seem more vague. Discourse Processes, 49, 523-538.

Levelt, W. J. M. (1983). Monitoring and Self-Repair in Speech. Cognition, 14(1), 41-104.

McQueen, J. M., \& Huettig, F. (2012). Changing only the probability that spoken words will be distorted changes how they are recognized. Journal of the Acoustical Society of America, 131(1), 509-517.

Niedzielski, N. (1999). The effect of social information on the perception of sociolinguistic variables. Journal of language and social psychology, 18(1), 62-85.

Pickering, M. J., \& Garrod, S. (2007). Do people use language production to make bpredictions during comprehension? Trends in cognitive sciences, 11(3), 105-110.

Sturt, P., Sanford, A. J., Stewart, A. J., \& Dawydiak, E. (2004). Linguistic focus and goodenough representations: an application of the change-detection paradigm. Psychonomic Bulletin \& Review, 11, 882-888.

Tanenhaus, M. K., Spivey-Knowlton, M. J., Eberhard, K. M., \& Sedivy, J. E. (1995). Integration of visual and linguistic information in spoken language comprehension. Science, 268, 1632-1634.

Traxler, M. J., Williams, R. S., Blozis, S. A., \& Morris, R. K. (2005). Working memory, animacy, and verb class in the processing of relative clauses. Journal of Memory and Language, 53, 204-224.

Uther, M., Knoll, M. A., \& Burnham, D. (2007). Do you speak E-NG-LI-SH? A comparison of foreigner-and infant-directed speech. Speech Communication, 49(1), 2-7.

Van Berkum, J., van den Brink, D., Tesink, C. M., Kos, M., \& Hagoort, P. (2008). The neural integration of speaker and message. Journal of Cognitive Neuroscience, 20, 580-591.

Veling, H., \& van Knippenberg, A. (2004). Remembering can cause inhibition: Retrieval-induced inhibition as cue independent process. Journal of Experimental Psychology. Learning, Memory, and Cognition, 30, 315-318. 